\title{
Unmet Needs for Informal Care Among People With Type 2 Diabetes in Rural Communities in Vietnam.
}

\section{Dan Wolf Meyrowitsch ( $\nabla$ dame@sund.ku.dk)}

University of Copenhagen https://orcid.org/0000-0003-1108-8987

\section{Jannie Nielsen}

GH: Emory University Hubert Department of Global Health

\section{Ib Christian Bygbjerg}

Department of Public Health, University of Copenhagen.

\section{Jens Søndergaard}

Department of Public Health, University of Southern Denmark.

\section{Diep Khong Thi}

Thai Binh University of Medicine and Pharmacy: Thai Binh Medical University

\section{Dieu Bui Thi Huyen}

Thai Binh University of Medicine and Pharmacy

Tine M. Gammeltoft

Department of Anthropology, University of Copenhagen

\section{Thanh Nguyen Duc}

Thai Binh University of Medicine and Pharmacy

\section{Research}

Keywords: Type-2 diabetes, informal care, unmet needs

Posted Date: January 5th, 2021

DOl: https://doi.org/10.21203/rs.3.rs-138524/v1

License: (c) (1) This work is licensed under a Creative Commons Attribution 4.0 International License. Read Full License 
Unmet Needs for Informal Care among People With Type 2 Diabetes in Rural Communities in Vietnam

Running title: Needs for Informal Diabetes Care in Vietnam

Dan Wolf Meyrowitsch, ${ }^{1}$ Jannie Nielsen, ${ }^{1,2}$ Ib Christian Bygbjerg, ${ }^{1}$ Jens Søndergaard, ${ }^{3}$ Diep Khong Thi, ${ }^{4}$ Dieu Bui Thi Huyen, ${ }^{4}$ Tine Gammeltoft, ${ }^{5} \&$ Thanh Nguyen Duc ${ }^{4}$

${ }^{1}$ Global Health Section, Department of Public Health, University of Copenhagen, Øster Farimagsgade 5, DK-1014, Copenhagen K, Denmark

${ }^{2}$ Emory Global Diabetes Research Cter, Hubert Department of Global Health, Rollins School of Global Health, Emory University, Atlanta, GA, USA

${ }^{3}$ Research Unit for General Practice, Department of Public Health, University of Southern Denmark, Denmark

${ }^{4}$ Thai Binh University of Medicine and Pharmacy, 373 Ly Bon Street, Thai Binh city, Thai Binh, Viet Nam.

${ }^{5}$ Department of Anthropology, University of Copenhagen, Øster Farimagsgade 5, DK-1353 Copenhagen K, Denmark

\section{Contact details for all authors}

Dan Wolf Meyrowitsch: PhD, Associate Professor, Head of research, Global Health Section, Department of Public Health, University of Copenhagen, Øster Farimagsgade 5, DK-1014, Copenhagen K, Denmark. Tel: +45 60604386, Fax: + 45 35327487, E-mail: dame@ sund.ku.dk

Jannie Nielsen: PhD, Assistant Professor, Emory Global Diabetes Research Cter, Hubert Department of Global Health, Rollins School of Global Health, Emory University, Atlanta, GA, USA. Tel: +45 22770909, E-mail: jannie.nielsen@emory.edu

Ib Christian Bygbjerg: Professor, Global Health Section, Department of Public Health, University of Copenhagen, Øster Farimagsgade 5, DK-1014, Copenhagen K, Denmark. Tel: +45 35327835, Fax: + 45 35327487, E-mail: iby@sund.ku.dk

Jens Søndergaard: Professor, Research Unit for General Practice, Department of Public Health, University of Southern Denmark, Denmark. Tel: +45 31372266, Fax: +45 6550 109, E-mail: jsoendergaard@ health.sdu.dk

Diep Khong Thi: Msc, Thai Binh University of Medicine and Pharmacy, 373 Ly Bon Street, Thai Binh city, Thai Binh, Viet Nam. Tel: +84 985546327, Fax:+84 22733847509, E-mail: diepkhongtbmu@gmail.com

Dieu Bui Thi Huyen: Msc, Thai Binh University of Medicine and Pharmacy, 373 Ly Bon Street, Thai Binh city, Thai Binh, Viet Nam. Tel: +84 889581410, Fax: +84 22733847509, E-mail:

huyendieu1410@gmail.com

Tine M. Gammeltoft: Professor, Department of Anthropology, University of Copenhagen, Øster Farimagsgade 5, DK-1353 Copenhagen K, Denmark. Tel: +4535323474, Fax: +45 35323465, email.: tine.gammeltoft@anthro.ku.dk

Thanh Nguyen Duc: PhD, Thai Binh University of Medicine and Pharmacy, 373 Ly Bon Street, Thai Binh city, Thai Binh, Viet Nam. Tel: +84 912357575; Fax: +84 2273847509; Email: thanhnd@ tbump.edu.vn

Word count: 3706 (exluding references)

Number of tables and figures: 3 
Abstract

\section{BACKGROUND}

From a diabetes management perspective, informal care has proven at least as important as care

from health professionals when targeting poorly controlled diabetes. The objective of this study was to identify determinants associated with unmet needs for informal support among people with type2 diabetes in rural communities of Vietnam.

\section{METHODS}

A cross-sectional survey was conducted from December 2018 to February 2019 in a rural area of northern Vietnam. From 2 districts in Thai Binh Province, Vietnam, 806 people with type-2 diabetes participated in a survey designed to assess who were their most important informal caregivers (ICGs) and to measure the association between demographic and socio-economic predictors and unmet needs of informal support of relevance for diabetes self-care using bivariate and multivariate analyses.

\section{RESULTS}

71 The spouse was reported as the most important ICG (62.9\%) followed by a daughter or son (28.4\%). $32.0 \%$ of the people with type-2 diabetes reported at least one type of unmet need for informal support. The most commonly reported unmet needs of informal care were: transport to health facilities and company when seeking formal care (20.5\%), financial support related to costs of diabetes self-management (18.5\%), and reminders to engage in physical exercise $(14.5 \%)$. 
1.25-11.52) and those being unemployed as compared to being retired (OR=2.85; CI95\%: 1.61 -

79

80

81

82

83

84

85

86

87

88

89

90

91

92

93

94

95

96

97

98

99

100
5.05).

\section{CONCLUSIONS}

Almost one-third of people with type-2 diabetes reported at least one type of unmet need for informal care. These findings provide a basis for development of new modalities for strengthening support provided by ICGs in rural communities in Vietnam and in other low- and middle-income countries.

Keywords: Type-2 diabetes, informal care, unmet needs.

\section{INTRODUCTION}

The anticipated increase in diabetes-related disease burden and a similar increase in burden of other chronic diseases call for new and innovative approaches to treatment and care. The traditional models of health service provision are under transformation and it has recently been emphasized that it is of crucial importance that all types of care givers and volunteers, who engage in health promotion, disease prevention, treatment, and rehabilitation, play an effective role in provision of primary health care (1-3). Particularly in resource constrained communities undergoing rapid transition of diseases patterns, informal health support should be assessed and considered as a modality for improving quality of life for both individuals living with chronic disease and their families. 
101 Diabetes self-management is regarded as an essential component in daily diabetes care concerning, 102 for example, diet, physical activity and medications (4). In this context, social interactions and 103 support from non-medical staff play a vital role among people living with disease (5), and this type 104 of informal care can potentially reduce diabetes-related distress and improve glycemic control (6). 105 Specifically, informal caregivers (ICGs), such as family members, friends, and neighbors, can play 106 an important role in self-care for people with type-2 diabetes by offering support related to issues 107 such as visits to health facilities, provision of medication, physical activity, and emotional needs 108 and thereby result in improved health outcomes over time $(7,8)$.

110 Persons with type-2 diabetes perceive family behaviors as being supportive for diabetes self111 management, as a barrier to diabetes self-management, or as equivocal behaviors with the potential 112 to both support and impede diabetes self-management (9-12).

114 The vast majority of studies on ICGs and diabetes self-management have been carried out in high115 income countries and, with few exceptions, focus solely on the positive health effects of informal 116 care. Very few studies have assessed the needs of informal care of relevance for self-management expressed by the persons with type- 2 diabetes themselves in low- and middle-income countries

118 (LMICs) undergoing rapid epidemiological shifts in patterns of disease, despite strong projections 119 that predict that the future major increase in numbers will take place in these LMICs (13).

121 Knowledge about unmet needs for informal support among persons with type-2 diabetes is of crucial importance for informing development of interventions aimed at bridging the gap between community members and resource constrained health systems. Therefore, a cross-sectional survey

124 was conducted in order to measure the occurrence and identify predictors of unmet needs for 
125 informal support among persons with type-2 diabetes in rural communities of Thai Binh Province, northern Vietnam.

\section{METHODS}

130 Study design

131 A questionnaire-based cross-sectional survey was conducted from December 2018 to February 2019 in a rural area of northern Vietnam.

134 Setting and recruitment of persons with type-2 diabetes

135 The study was carried out in Thai Binh Province. Located 100 kilometers Southeast of Hanoi city, it 136 covers an area of $1,542 \mathrm{~km}^{2}$ and has a total population of approximately 1.86 million people. It is 137 divided into 8 districts and 284 communes. Two rural districts, Quynh Phu District in the northern

138 part and Vu Thu District in the southern part of the district, were purposively selected for the 139 project based on district hospital records showing that these two districts had the highest number of

140 people diagnosed with diabetes. There are two district hospitals in Quynh Phu District and one

141 district hospital in $\mathrm{Vu}$ Thu District. Based on lists of people who received treatment for diabetes, 142 that were available at these district hospitals, all communities were ranked according to the number 143 of such persons in each of the two districts. In each district, among the communities with the 144 highest number of persons with type-2 diabetes, two communities with the closest proximity to the 145 district hospital and two communities located farthest from the district hospital were selected for the 146 study. Hence, a total of 8 communities were purposively selected for the study. For the analytic 147 sample, people who were diagnosed with diabetes before age 40 years were excluded. 
149 The sampling frame, that is, the list of individuals in the 8 communes treated for diabetes at the 150 district hospitals, included a total of 963 individuals. All were invited to participate in the study. 151 Among these, $37(3.8 \%)$ refused to participate, $78(8.1 \%)$ did not stay at the address reported to the

152 hospital, or had moved away by the period of data collection. Furthermore, 42 individuals were

153 excluded from the analysis since they reported that they had been diagnosed with diabetes prior to

154 the age of 40 years or they did not remember when they had been diagnosed. Hence, a total of 806 persons were included in the study ( $83.7 \%$ of individuals in the sampling frame).

Interviews

From each health station in the 8 selected communes, two health workers were trained to administer the questionnaires and to conduct the interviews. The interviewers were trained in a 2-day workshop followed by field-based training and counselling. As part of the training, the interviewers participated in the pilot testing and subsequent revisions of the questionnaires.

All potential interviewees were contacted by mobile phone calls or by personal visits to request their participation and, if they agreed, fix the date and time of the interview. The interviews took place in their homes. Prior to the interview, the aim and outline of the research project were explained verbally and in writing. Those who agreed to participate signed a consent form.

Questionnaires

169 Each interviewee was asked about who they perceived as their most important ICG of relevance for their diabetes self-management. For descriptive analyses, ICGs were categorized as: 1) spouse; 2)

171 daughter or son; 3) other relative (e.g. parents, brother, sister, son-in-law, daughter-in-law); and 4) non-relative (e.g. other person with diabetes, friend, neighbor). 
Outcome variable

175 Unmet needs for informal care were based on six questions regarding: 1) transport to clinic or

176 hospital when seeking care related to type-2 diabetes and being accompanied by an ICG; 2)

177 purchase and preparation of food of relevance for type-2 diabetes diet; 3) reminder to take

178 medication for type-2 diabetes; 4) reminder to engage in physical exercise; 5) emotional support in

179 relation to type-2 diabetes; and 6) financial support of specific importance for self-management of

180 diabetes. The answering options for these questions were: 1) inadequate level of care; 2) adequate

181 level of care; and 3) high level of care. For each dimension of informal care, 'inadequate level of

182 care' was used to define a high level of unmet need for informal care, whereas an 'adequate level of

183 support' or 'high level of support' was used to define a low level of unmet need for informal care.

184 An overall unmet need of informal care was defined as at least two answers of 'inadequate level of

185 care' to the six questions mentioned above.

186

187 Exposure variables

188 Possible predictors, demographic and socio-economic characteristics, and self-reported status of 189 physical health, were selected a priori based on assumptions that these were typical determinants for

190 unmet needs related to management of diabetes, and comprised possible modalities for

191 interventions targeting diabetes. The demographic and socio-economic predictors included sex, age,

192 marital status, size of household, occupational status, and economic status of the household.

194 For the statistical analysis of the association between exposure variables and unmet needs, age was

195 categorized into four age groups: 1) 40-49 years; 2) 50-59 years; 3) 60-69 years; and 4) $\geq 70$ years).

196 Marital status was categorized into two groups: 1) living with spouse; and 2) single, divorced, 
widowed, or living separate from spouse. Size of household was categorized into: 1) 1 member

198 (living alone); 2) 2 members; 3) 3-4 members; and 4) $\geq 5$ members. Occupational status was

199 categorized as: 1) unemployed; 2) farmer; 3) being employed in small trade business, as a worker,

200 government employee or in a private company; and 4) retired. Self-reported economic status of the

201 household of the person with type-2 diabetes was categorized as: 1) poor; 2) medium; and 3)

202 wealthy. Self-reported level of physical health was categorized into: 1) poor; 2) fair; and 3) good.

204 Statistical analysis

205 Data were double-entered in EPI-DATA 3.1 for quality control and errors were subsequently 206 corrected. SPSS software package (IBM SPSS Statistics, version 25, 2017) was used for data 207 analysis. Descriptive data are presented in frequency tables. Bivariate and multivariate analyses 208 were used to measure the associations between exposure variables and overall unmet need for 209 informal support. Results were reported as odds ratios (ORs) and 95\% confidence intervals around 210 the respective ORs. Predictors in the bivariate analyses, which were associated with unmet needs at 211 a 5\% level or lower, were included in the multivariate analysis.

RESULTS

216 Sample characteristics

217 Characteristics of the persons with type-2 diabetes are summarized in Table 1. Approximately half 218 of them were females (52.7\%). Almost one-third were aged 70 years or older $(30.5 \%)$, and the 219 majority lived with a spouse (73.6\%). One out of ten respondents lived alone (11.0\%), whereas half 220 of them lived in a household with more than two members (49.5\%). One out of nine described their 
221 household as poor (11.8\%), whereas the majority described their household as having a medium 222 level of wealth (79.2\%). More than one-third of the persons with type-2 diabetes (37.5\%) reported 223 their physical health as being poor.

Informal caregivers

226 Irrespective of whether they lived with a spouse or not, a spouse was reported as the most important 227 ICG (62.9\%) followed by a daughter or son (28.4\%). Among those who did not live with a spouse 228 or did not have a spouse, a son or daughter was reported as the most important ICG (67.6\%) 229 followed by another relative (25.8\%), whereas a smaller proportion $(6.6 \%)$ indicated a non-relative 230 as the most important ICG.

Unmet needs for informal care

Overall, $32.0 \%$ of the sample reported at least one type of unmet need for informal care. Overall, $11.8 \%, 7.1 \%$, and $13.1 \%$ reported 1 type, 2 types and 3-6 types of unmet need for informal care, respectively.

The occurrence of unmet need for informal care for each of the five dimensions of care is shown in Table 2. The most commonly reported unmet need was transport to clinic or hospital and company 239 during the visit (20.5\%), followed by an unmet need for financial support related to costs of 240 diabetes self-management (18.5\%) and an unmet need for reminders to engage in physical exercise 241 (14.5\%). Across all six dimensions of care, women and those who lived alone had relatively high proportions of unmet need. Those who reported poor physical health presented with the highest proportions of unmet need for informal care across all six dimensions of support. 
245 The lowest proportions of unmet needs for informal care were observed for the dimension of 246 emotional support among men (1.6\%) and for those persons who reported good physical health $247(1.9 \%)$.

A separate sub-analysis was performed among those who lived alone. In this group, the highest proportions of unmet needs for informal care were transport to health facility and financial support (46.1\% and $40.4 \%$, respectively).

The results of the analysis of the association between selected predictors and overall level of unmet need for informal care are presented in Table 3. The results of the multivariate analysis showed that living alone as compared to living in a household with five members had the highest odds ratio for overall level of unmet need for informal care $(\mathrm{OR}=4.41$; CI95\%: 2.19-8.88) followed by being poor as compared to being wealthy $(\mathrm{OR}=3.79 ; \mathrm{CI} 95 \%: 1.25-11.52)$ and being unemployed as compared to being retired $(\mathrm{OR}=2.85$; CI95\%: 1.61-5.05). Lastly, poor level of self-reported physical health as compared to good health was associated with a high risk of unmet need (AOR=2.31; CI95\%: 1.055.08).

\section{DISCUSSION}

265 In the present study, we assessed who were the most important ICGs, and the associations between 266 demographic and socio-economic predictors and unmet needs of informal care of relevance for 267 diabetes self-management in a rural population in Vietnam. The spouse was the most important ICG 268 for diabetes self-management, and this finding is in accordance with other studies $(8,14,15)$. 
Among those persons with type-2 diabetes who did not live with a spouse, the most important ICG was a son or a daughter.

The findings in the present study suggest that for most persons with type- 2 diabetes who experience unmet needs of informal care, the request for support is restricted to one or two specific dimensions. The needs for assistance and transport when visiting health facilities and financial support of relevance for self-management of diabetes were expressed by approximately one out of five persons.

Across all six dimensions of support, women were more likely to report an unmet need of informal support than men. Previous studies have shown that women had a higher risk to present with diabetes-related distress than men (16-18). In Vietnam, women are often separated from their natal families after marriage, where after they commonly move to the household of their husband's family (19). This may result in gender-specific barriers in sharing chronic disease-related difficulties in life with their family (20). Furthermore, in Vietnam, women often bear the main responsibility for housework and for taking care of children and elderly members of their household (21). These responsibilities in combination with other sociocultural factors may result in a lower level of informal care offered to women due to gendered moral expectations and household division of labor.

Persons with type-2 diabetes living alone as compared to living in a larger household, being unemployed or a farmer as compared to being retired, having poor physical health as compared to good health were associated with a high level of unmet need for informal support for diabetes management. Specific needs for informal care and barriers for access to health care among those 
who lived alone have been reported in other studies $(22,23)$. Although women, as previously mentioned, were more likely to report an unmet need for a specific type of support than men, results of the multivariate analysis showed no gender differences when using an aggregated measure for an overall unmet need for informal care.

The present study included a total of 806 persons living with type-2 diabetes, which allowed detailed analyses of determinants for unmet need of informal care of relevance for diabetes related self-management. However, the study has some limitations related to design, analysis, and validity of results. Since persons were recruited using a sampling frame of individuals treated with diabetes at the district hospitals, community members being referred to and treated at regional hospitals were not included as study participants. Therefore, it is not known whether the group of respondents included in the present study is representative of persons with type- 2 diabetes in this population. In Vietman, those treated at a regional hospital typically present with more severe disease as compared to those treated at the district hospitals. This would most likely result in an underestimation of the prevalence of persons with type-2 diabetes with unmet needs for informal care. Furthermore, the focus on unmet needs for informal care addressed six pre-defined dimensions of care, and it is likely that there are other types of needs of importance for diabetes self-management which have not been covered in the present study, e.g. a specific need for interpretation of advice and counselling offered by health providers.

313 Efforts were made to pilot test and modify the questionnaire to match the local context with regards to gender issues, terminology, and cultural norms and values. However, there was still a risk of responder bias, specifically regarding the motivation to disclose that there are daily problems and challenges related to a life with a chronic disease. On the condition that responder bias was equally 
distributed between the groups being compared, this type of non-differential misclassification of the outcome would not have affected the observed associations.

Although efforts were made to include the most relevant predictors for unmet support, residual confounding, e.g. alternative measures for severity of disease, size and character of social network, individual variations in resilience, co-morbidity, and spouses with chronic disease, may also have influenced the observed statistical associations; though it is difficult to comprehend how specific types of confounding would have affected the findings.

It was not assessed whether persons with type- 2 diabetes perceived that ICGs impede diabetes selfmanagement as reported in other studies from high-income countries (9-11). Future research needs to be done to assess whether certain types of informal care tend to challenge or obstruct diabetes related self-management.

In the present study, it is not known whether a high level of unmet need for informal care in this population actually results in a higher risk of negative diabetes related health outcomes as compared to individuals with lower levels of unmet need. However, results from several studies, with the majority performed in high-income countries, indicate that supportive social support measures are associated with improved health among persons with type-2 diabetes $(7,24,25)$, specifically with regard to self-management related to diet, exercise, blood glucose testing, HbA1c levels, and adherence to medication $(8,10,26)$. Another study has demonstrated that greater closeness with informal care partner is a significant predictor for medical adherence, higher fruit/vegetable intake, and lower diabetes-related distress (27). 
341 These findings suggest a potential for including spouses in a systematic approach to improve 342 quality of life and self-management of disease among persons with type-2 diabetes in rural 343 communities of Vietnam and other LMICs.

\section{CONCLUSIONS}

347 In conclusion, the results of the present study performed in rural communities in Vietnam provide a 348 basis for improving care and support from ICGs, as reported in other studies $(11,12,24,26,28)$. 349 For individuals with diabetes who live with a spouse or are single/widowed, the results suggest that 350 there are obvious advantages in including the spouse or daughter/son, respectively, in counselling 351 and training activities which will support and improve self-management. Persons with type-2 352 diabetes who live alone, are unemployed, poor, or with bad physical health should specifically be 353 considered as highly vulnerable groups with a high level of unmet need for care addressing all six 354 dimensions of support. Results from the present study also suggest that women with diabetes have 355 specific needs for assistance with transport when visiting health facilities and for financial support 356 of relevance for their self-management of diabetes. Family members and health providers need to 357 be aware of the need for assistance and transport when barriers to self-management of diabetes and 358 should take action and improve support for transport and assist in reducing the individual economic 359 burdens related to a life with diabetes. Furtermore, this underpins the importance of future 360 educational programmes, with focus on diabetes self-management, to be tailored to target the 361 specific needs for informal care. 
363 In already overburdened resource-constrained health systems in LMICs, strengthening support and 364 health care offered by ICGs may appear as a cost-effective intervention which will result in 365 improved quality of life for both individuals living with disease and their family members.

367 List of abbreviations

368 ICG: Informal caregivers

369 LMICs: Low- and middle-income countries

370 OR: Odds ratio

373 Declarations

375 Ethics approval and consent to participate

376 The study followed ethical guidelines developed by CIOMS (Council for International Organization 377 of Medical Sciences). Both oral and written informed consent were obtained from all research 378 participants and confidentiality was guaranteed. Ethical approval of the project was granted by 379 the Ethics Committee for Biomedical Research of Thai Binh University of Medicine and Pharmacy 380 (decision no. 11/2018, 23rd November 2018).

382 Consent for publication

383 Not applicable.

385 Availability of data and material

386 The datasets during and/or analysed during the current study available from the corresponding 
author on reasonable request.

Competing interests

391 The authors declare that they have no competing interests.

$393 \quad$ Funding

394 The present study is part of the interdisciplinary research project, Living Together with Chronic 395 Disease: Informal Support for Diabetes Management in Vietnam (VALID) (17-M09-KU), funded by the Ministry of Foreign Affairs of Denmark. The research is conducted in collaboration with the Strategic Sector Cooperation project, Strengthening the Frontline Grassroots Health Worker: Prevention and Management of NCDs at the Primary Health Care Level, carried out by the 399 Ministries of Health of Denmark and Vietnam.

401 Authors' contributions

402 All authors contributed to the development of data collection tools and training of interviewers.

403 DKT and DHTB participated in data collection. DM analyzed the data. DM and NDT developed the 404 first version of the manuscript. All authors contributed to subsequent revisions and editing of the 405 manuscript.

407 Acknowledgements

408 We are grateful to the research assistants from Quynh Phu District and Vu Thu District who 409 conducted the interviews and we thank all staff at the Population Center and Community Health 
410 Centers in Thai Binh Province for their assistance. Specifically, we would like to thank all the 411 community members from Quynh Phu District and Vu Thu District who participated in the 412 interviews.

415 References

$417 \quad$ 1. WHO. Building the primary health care workforce of the 21st century. 2018.

418 2. Rosland AM, Piette JD, Choi H, Heisler M. Family and friend participation in primary 419 care visits of patients with diabetes or heart failure: patient and physician determinants and 420 experiences. Med Care. 2011;49(1):37-45.

$421 \quad 3 . \quad$ Pecchioni LL, Sparks L. Health information sources of individuals with cancer and 422 their family members. Health Commun. 2007;21(2):143-51.

424 Self-management Education and Support in Type 2 Diabetes. Diabetes Educ. 2017;43(1):40-53. 425 5. DiMatteo MR. Social support and patient adherence to medical treatment: a meta426 analysis. Health Psychol. 2004;23(2):207-18.

427 6. Lee AA, Piette JD, Heisler M, Janevic MR, Rosland AM. Diabetes self-management 428 and glycemic control: The role of autonomy support from informal health supporters. Health 429 Psychol. 2019;38(2):122-32.

$430 \quad$ 7. Nicklett EJ, Heisler ME, Spencer MS, Rosland AM. Direct social support and long-term 431 health among middle-aged and older adults with type 2 diabetes mellitus. J Gerontol B Psychol Sci 432 Soc Sci. 2013;68(6):933-43. 
434 and effects on diabetes self-care activities in Korean Americans with type 2 diabetes. Diabetes 435 Educ. 2012;38(1):77-85.

$4369 . \quad$ Mayberry LS, Osborn CY. Family support, medication adherence, and glycemic 437 control among adults with type 2 diabetes. Diabetes Care. 2012;35(6):1239-45.

438 10. Mayberry LS, Osborn CY. Family involvement is helpful and harmful to patients' self439 care and glycemic control. Patient Educ Couns. 2014;97(3):418-25.

440 11. Rosland AM, Heisler M, Choi HJ, Silveira MJ, Piette JD. Family influences on self441 management among functionally independent adults with diabetes or heart failure: do family 442 members hinder as much as they help? Chronic IIIn. 2010;6(1):22-33.

443 12. Vongmany J, Luckett T, Lam L, Phillips JL. Family behaviours that have an impact on 444 the self-management activities of adults living with Type 2 diabetes: a systematic review and 445 meta-synthesis. Diabet Med. 2018;35(2):184-94.

446 13. Federation ID. IDF Diabetes Atlas2019 [cited 2020 10th January 2020]. Available 447 from: https://www.diabetesatlas.org/en/.

448 14. Pesantes MA, Del Valle A, Diez-Canseco F, Bernabe-Ortiz A, Portocarrero J, Trujillo A, 449 et al. Family Support and Diabetes: Patient's Experiences From a Public Hospital in Peru. Qual 450 Health Res. 2018;28(12):1871-82.

$451 \quad$ 15. Gupta L, Khandelwal D, Lal PR, Gupta Y, Kalra S, Dutta D. Factors Determining the 452 Success of Therapeutic Lifestyle Interventions in Diabetes - Role of Partner and Family Support. Eur 453 Endocrinol. 2019;15(1):18-24. 

complex interplay between clinical and person-centered diabetes outcomes in the two genders. Health Qual Life Outcomes. 2017;15(1):41. psychological distress among diabetes mellitus adults in the Jilin province in China: a crosssectional study. PeerJ. 2017;5:e2869.

46018 Fisher L, Mullan JT, Skaff MM, Glasgow RE, Arean P, Hessler D. Predicting diabetes 461 distress in patients with Type 2 diabetes: a longitudinal study. Diabet Med. 2009;26(6):622-7. 46219. Nhi TT, Hanh NTT, Gammeltoft TM. Emotional violence and maternal mental health: 463 a qualitative study among women in northern Vietnam. BMC Womens Health. 2018;18(1):58. 20. Tho Tran N, Nguyen HTT, Nguyen HD, Ngo TV, Gammeltoft T, Rasch V, et al.

465 Emotional violence exerted by intimate partners and postnatal depressive symptoms among 466 women in Vietnam: A prospective cohort study. PLoS One. 2018;13(11):e0207108.

467 21. Gammeltoft T. Women's Bodies, Women's Worries: Health and Family Planning in a 468 Vietnamese Rural Commune: Routledge; 1 edition (December 14, 1998); 1998.

469 22. Miyawaki Y, Shimizu Y, Seto N. Classification of Support Needs for Elderly Outpatients 470 with Diabetes Who Live Alone. Can J Diabetes. 2016;40(1):43-9.

471 23. Sakai R, Hashimoto Y, Hamaguchi M, Ushigome E, Okamura T, Majima S, et al. Living 472 alone is associated with visit-to-visit HbA1c variability in men but not in women in people with 473 type 2 diabetes: KAMOGAWA-DM cohort study. Endocr J. 2020.

474 24. Epple C, Wright AL, Joish VN, Bauer M. The role of active family nutritional support in 475 Navajos' type 2 diabetes metabolic control. Diabetes Care. 2003;26(10):2829-34. 
$47625 . \quad$ Park PH, Wambui CK, Atieno S, Egger JR, Misoi L, Nyabundi JS, et al. Improving 477 Diabetes Management and Cardiovascular Risk Factors Through Peer-Led Self-management 478 Support Groups in Western Kenya. Diabetes Care. 2015;38(8):e110-1.

479 26. Bouldin ED, Trivedi RB, Reiber GE, Rosland AM, Silverman JB, Krieger J, et al. 480 Associations between having an informal caregiver, social support, and self-care among low481 income adults with poorly controlled diabetes. Chronic Illn. 2017;13(4):239-50.

482 27. Mayberry LS, Piette JD, Lee AA, Aikens JE. Out-of-home informal support important 483 for medication adherence, diabetes distress, hemoglobin A1c among adults with type 2 diabetes. J 484 Behav Med. 2019;42(3):493-501.

485 28. Lee AA, Piette JD, Heisler M, Rosland AM. Diabetes Distress and Glycemic Control: 486 The Buffering Effect of Autonomy Support From Important Family Members and Friends. Diabetes 487 Care. 2018;41(6):1157-63. 


\section{Supplementary Files}

This is a list of supplementary files associated with this preprint. Click to download.

- UnmetneedsforinformalcareTables.pdf 